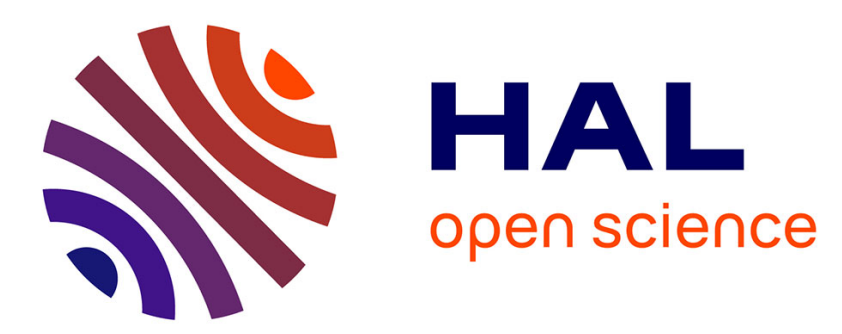

\title{
Les pressions anthropiques, les mesures de protection et les défis de gestion participative en Guadeloupe et à la Martinique
}

Michel Desse

\section{- To cite this version:}

Michel Desse. Les pressions anthropiques, les mesures de protection et les défis de gestion participative en Guadeloupe et à la Martinique. Chouinard Omer, Batzan Juan, Vanderlingen Jean-Paul. zones côtières et changement climatique, Presses de l'Université du Québec, 2011, 978-2-7605-3188-8. hal01172199

\author{
HAL Id: hal-01172199 \\ https://hal.science/hal-01172199
}

Submitted on 7 Jul 2015

HAL is a multi-disciplinary open access archive for the deposit and dissemination of scientific research documents, whether they are published or not. The documents may come from teaching and research institutions in France or abroad, or from public or private research centers.
L'archive ouverte pluridisciplinaire $\mathbf{H A L}$, est destinée au dépôt et à la diffusion de documents scientifiques de niveau recherche, publiés ou non, émanant des établissements d'enseignement et de recherche français ou étrangers, des laboratoires publics ou privés. 
DESSE M, (2011). Les pressions anthropiques, les mesures de protection et les défis de gestion participative en Guadeloupe et à la Martinique. In zones côtières et changement climatique. Sous la direction d'O Chouinard, J Baztan et J-P Vanderlinden. Presses de l'Université du Québec.pp165-166.

\section{Publication aux Presses Universitaires du Québec}

\section{Pressions anthropiques, mesures de protection et défis de gestion participative en Guadeloupe et à la Martinique.}

\section{Michel Desse}

Professeur des Universités

ICOTEM

Université de Poitiers.

\section{Résumé}

Les littoraux de Guadeloupe et de Martinique connaissent des pressions anthropiques nombreuses et variées du fait de la mise en tourisme et surtout de la transformation des modes de vie entraînant la requalification sociale et fonctionnelle des zones côtières. Si de larges portions littorales sont protégées grâce à la mise en réserve terrestre ou marine, ou par le biais des Parc Naturels, se pose la question de la gestion des zones littorales densément peuplées. Nous présenterons certaines initiatives de GIZC qui montrent les difficultés à dynamiser les différents acteurs et à mettre en place une gestion participative.

Mots clés : Guadeloupe, Martinique, GIZC, littoralisation, gestion, aménagement.

\section{Summary}

The coastlines of the islands of Guadeloupe and Martinique have been exposed to variegated forms of anthropic pressure due to the development of tourism and above all to the transformation of the islanders' ways of life, which 
have in turn required a parallel evolution of the social and functional uses of coastal areas. Though large portions of the coastlines have been preserved because they were public property or they belonged to natural reserves, the management of densely populated coastal zones has remained an issue. I shall present here several integrated coastal management initiatives which highlight both the inertia of various contributors to coastal management and the problems associated with public participation in such an enterprise.

Key Words: Guadeloupe, Martinique, integrated coastal zone management,

Si dans les années 1970, on quittait les mornes et les petits villages agricoles pour les villes capitales et les aires périurbaines, depuis la fin des années 1980 de nouvelles dynamiques apparaissent en faveur des communes littorales et surtout balnéaires. Ainsi, les littoraux de Guadeloupe et de Martinique connaissent des pressions anthropiques nombreuses et variées du fait de la mise en tourisme et de la transformation des modes de vie et des regards portés sur la mer. Ceci est renforcé par les municipalités qui réorganisent leur front de mer, déplaçant les populations les plus pauvres afin de les protéger des aléas cycloniques. Dans ces conditions, les pressions foncières sont fortes entraînant la requalification sociale et fonctionnelle des zones côtières.

Depuis quelques décennies de larges portions littorales sont protégées grâce à la mise en réserve terrestre ou marine, ou par le biais des parcs naturels, se pose la question de la gestion des zones littorales densément peuplées. Nous présenterons certaines initiatives de GIZC qui montrent les difficultés à dynamiser les différents acteurs et à mettre en place une gestion participative. En effet, ces nouvelles populations littorales ont parfois des liens à la mer très réduits et sont peu impliquées à la vie locale. L'action des acteurs politique est elle aussi freinée par la forte pression foncière et la spéculation qui empêchent parfois la force et la cohérence des politiques publiques sur le long terme.

\section{La requalification territoriale des littoraux antillais}

Dès la prise de possession de la Guadeloupe et de la Martinique, les littoraux accueillent les premières bourgades et les premières fortifications. Très rapidement, avec le développement de l'économie de plantation, les îles se détournent partiellement de la mer pour adopter les valeurs de la terre reposant sur les cultures d'exportation, l'habitation et le bien foncier. 


\subsection{Du glacis militaire au réceptacle de l'exode rural}

Dans les îles françaises, dès 1674, la bande côtière des « 50 pas du Roi » qui appartient au domaine privé de la couronne est instituée en réserve, libre de passage afin d'y construire des fortifications. Cependant dès l'origine, les artisans (pêcheurs, maçons et charpentiers) peuvent s’y installer. À partir de 1827 , les « 50 pas du Roi » entrent dans le domaine public, devenant inaliénables. Avec la disparition de la monarchie, les «50 pas du Roi » deviennent les « 50 pas géométriques » et s'étendent sur $81,20 \mathrm{~m}$.

À la suite de l'émancipation en 1848 mettant fin à l'esclavage et surtout entre 1930 et 1970, les populations indigentes investissent les littoraux, surtout à proximité des agglomérations. Cette appropriation d'un territoire ouvert, n'est pas revendiquée par l'Etat et est même encouragée par les municipalités qui manquent de réserves foncières. À Fort-de-France, Aimé Cézaire a ainsi soutenu les habitants des quartiers d'habitat spontané de Volga Plage et de Texaco, qui ont construit sur les «50 pas géométriques ». A Pointe-à-Pitre, le quartier du Carénage s'étire entre la friche industrielle de l'ancienne usine sucrière Darbousier et la Marina (cliché $\mathrm{n}^{\circ} 2$ ). Cette littoralisation des personnes indigentes touche aussi les bourgs littoraux dont les façades littorales s'étirent. Au Prêcheur, à la Martinique, $70 \%$ de la population vit sur le domaine public maritime. Aux Anses d'Arlets comme à Deshaies en Guadeloupe, les marins pêcheurs occupaient le haut de plage et la limite de la cour se situait à quatre ou cinq mètres de la mer (cliché $n^{\circ} 1$ ). Ailleurs les pêcheurs constituent des quartiers originaux, s'étirant le long du rivage de part et d'autre des centres-bourgs (Saint-François en Guadeloupe). Parfois les populations halieutiques construisent des hameaux distincts des centre-bourg comme à Baille-Argent, à Rifflet en Côte-Sous-le-Vent en Guadeloupe. D'autres s'installent en fonction de leur territoire de pêche, expliquant l'habitat sur les pointes ou en arrière des mangroves ainsi que les remises à matériel et petites maisons sur les îlets.

Depuis 1996, ces quartiers littoraux qui ont accueilli le trop plein des campagnes sont reconnus par l'état. La loi du 30 décembre 1996 relative à l'aménagement, la protection et la mise en valeur de la zone dite des « 50 pas géométriques » dans les départements d'OutreMer conforte la loi littorale de 1986 en limitant les constructions aux zones urbanisées ou d'habitat diffus. Les occupants doivent prouver que la parcelle était construite avant le $1^{\text {er }}$ janvier 1955 et occupée à usage d'habitation principale ou de bâtiment professionnel pour obtenir une cession à titre onéreux en fonction de la valeur vénale du terrain nu. La superficie du terrain est plafonnée à $500 \mathrm{~m}^{2}$. Dans le cas d'un bâtiment à usage professionnel, la superficie du terrain ne peut excéder plus de $50 \%$ de celle occupée par le bâtiment et les installations au sol. La commission départementale de vérification des titres valide les titres 
de propriété antérieurs au décret du 30 juin 1955 et établit les droits de propriété réels ou de jouissance si la personne requérante n'en possède pas avant le $1^{\mathrm{er}}$ janvier 1995. D'après la Direction Départementale de l'Equipement de la Martinique, près de 62000 personnes seraient concernées et occuperaient le Domaine Public Maritime. Quand les conditions sanitaires sont mauvaises et quand l'exposition aux risques naturels sont trop fortes, ces populations littorales sont aujourd'hui réinstallées en arrière. Le front de mer devient alors un lieu récréatif, ou occupé par des restaurants et magasins liés au tourisme, ou parfois donne lieu à la construction de programmes résidentiels de standing.

\subsection{Les fermetures ponctuelles}

À partir des années 1970, les rivages constituent une interface ouverte aux impulsions locales (habitat spontané), ou exogènes (hôtels internationaux, zones portuaires, zones industrielles) et deviennent multifonctionnels. Ces aménagements en chapelet tendent alors à cloisonner le littoral.

Afin de développer le tourisme international, les différents plans de développement économique entre 1956 et 1970, encouragent la construction de grands hôtels de luxe de grande capacité. Ces hôtels se regroupent autour d'équipements structurants (casino, ports de plaisance, practices de golf) aux Trois-Ilets à la Martinique, Le Gosier, Sainte-Anne et SaintFrançois en Guadeloupe. Depuis 1986, les lois de défiscalisation ont amplifié les constructions dans ces espaces touristiques qui apparaissent ainsi comme des lieux très attractifs pour la grande hôtellerie mais aussi pour la petite hôtellerie de luxe, les résidences locatives et la location de villas ou d'appartements par les particuliers. Ces quartiers animés concentrent les entreprises de location de voitures, de voiliers, les magasins de mode, les restaurants. Ce dynamisme attire les insulaires qui y construisent entraînant une mixité des habitats résidentiels et à vocation touristique (cliché $\left.n^{\circ} 4\right)$.

Avec la mise en place de la Départementalisation, les Zones Industrialo- Portuaires ont connu un essor important et occupent des portions importantes de fronts de mer urbains. Le port autonome de Pointe-à-Pitre occupe toujours les anciens quais qui bordent la ville, mais 90\% du commerce maritime transitent par le port de Jarry qui dispose de 17 postes répartis sur 2300 mètres de quai. La zone industrielle et commerciale portuaire couvre 100 hectares sur deux sites au cœur de la zone industrielle et commerciale de Jarry. C'est le premier pôle économique de l'île qui regroupe plus de 800 entreprises. De manière générale les zones 
d'activités, les ZIP, certaines pistes d'aéroport, les routes utilisent les terrains plats de bord de mer.

\subsection{La forte spéculation foncière récente}

Si jusqu'aux années 1970, la littoralisation concerne des espaces contigües aux bourgs et quelques cases isolées, à partir des années 1980, les constructions suivent le rivage ou s'étagent sur les hauteurs afin de bénéficier de la vue sur la mer (cliché $n^{\circ} 3$ ). Les lois de défiscalisation ont entraîné le développement de programmes immobiliers de maisons ou d'immeubles à des fins touristiques mais aussi résidentielles. Le regard des Antillais sur la mer s'est transformé et les pratiques nautiques se sont intensifiées. Voir la mer depuis les lotissements belvédères sur les pentes des mornes, comme à proximité du littoral devient un marqueur d'un certain niveau de vie, une valeur ajoutée, signe évident de la réussite sociale.

Ainsi de nouveaux quartiers se forment accueillant, Guadeloupéens et Martiniquais, mais aussi des Métropolitains en poste ou désirant passer leur retraite sous les tropiques, auxquels se mêlent les migrants de retour des diasporas insulaires (cliché $\mathrm{n}^{\circ} 4$ ). Ces communes touristiques et résidentielles connaissent de fortes dynamiques démographiques. Dans les communes situées au sud de la Martinique, les variations de population entre 1999 et 2008 s'élèvent à $11 \%$ à Sainte-Luce, $12 \%$ à Sainte-Anne, $18 \%$ au Marin, à 37,6\% aux Trois-Ilets et à 41,5\% au Diamant.

Depuis, la loi littorale de 1986, puis l'application des différents plans d'urbanisme et d'aménagement, la pression foncière s'accentue. En effet, le choix de permettre la construction de maison individuelle, très consommateur d'espace, dans le cadre insulaire étroit, nécessitant par ailleurs, de conserver de tout habitat, certains secteurs remarquables, agricoles ou soumis à des risques naturels, renforce la pression foncière sur certains secteurs et stimule la spéculation foncière. Ce mouvement de littoralisation entraîne ainsi une forte ségrégation, et génère une fragmentation sociale. Parfois des villas de luxe jouxtent des quartiers très modestes. Ces nouveaux arrivants accentuent les distorsions sociales par leur niveau de vie plus élevé et leur mode de vie hédoniste. Tout ceci contribue à creuser l'écart des revenus et finalement à juxtaposer, voire à faire s'entrechoquer des modes de vie qui s'opposent. Ainsi $1000 \mathrm{~m}^{2}$ atteignent 100000 euros dans les communes touristiques de Martinique et de Guadeloupe contre 45000 euros dans des communes rurales de la côte au vent. 
2. Les acteurs de la gestion du littoral

Les professionnels du littoral comme les touristes ou ceux qui y pratiquent un loisir sont souvent utilisateurs des écosystèmes et participent plus ou moins consciemment à leur dégradation, mais ils engagent aussi des actions pour les valoriser et participent parfois à leur régénérescence.

2.1. Des utilisateurs qui participent aussi à la meilleure gestion du littoral.

Les marins-pêcheurs ressentent les impacts des dégradations écologiques et la nécessité de la gestion. A la Martinique, le Comité des pêches, relayé par les associations locales des marins-pêcheurs, a mis en place des zones de cantonnement de pêche, où toute pratique est interdite durant plusieurs années afin de reconstituer les stocks. Le Comité des pêches demande régulièrement le concours des Affaires Maritimes pour détruire les nasses sur les zones ainsi protégées. Les Comités locaux des pêches participent à la mise en service des dispositifs de concentration du poisson D.C.P et à leur entretien afin de soulager la pression halieutique sur les eaux côtières. Les pêcheurs se mobilisent aussi contre la dégradation des écosystèmes côtiers.

Les professionnels du tourisme sont à la fois agents destructeurs et acteurs d'une meilleure protection des écosystèmes littoraux. Après une première période d'exploitation de la nature (les sites de plongée) qui a conduit à de nombreuses destructions, la filière de l'écotourisme et du tourisme durable tend à jouer un rôle dans la protection et la didactique environnementale. La mise en tourisme contribue aussi à changer le regard des insulaires sur les écosystèmes littoraux (les mangroves) qui deviennent des supports économiques. Il en est de même pour les îlets qui font aujourd'hui l'objet de mesures de protection. Ainsi les entreprises de plongée et les compagnies qui assurent la découverte des fonds coralliens en bateaux à fond de verre font preuve de didactique auprès des visiteurs qui ne sont pas tous touristes, pour louer les mérites de la réserve marine, de la zone de cantonnement de pêche, pour expliquer les bons gestes (ne pas casser les coraux, palmer en douceur...) et transmettre des connaissances scientifiques. Les pêcheurs qui promènent les touristes sur leurs canots sont aussi des diffuseurs de connaissances, parfois avec une certaine naïveté mais qui ont le mérite de venir d'hommes du métier et écoutés alors comme une valeur sûre.

Les hôtels participent aussi à la gestion du littoral, tout au moins pour les parties d'hôtel qui contribuent au décor : les jardins, le parc arboré, l'intégration architecturale, la plage. Il s'agit alors d'une gestion/mise en scène qui peut parfois être réussie. Enfin, les spots télévisés 
et les panneaux publicitaires incitant les touristes à respecter l'environnement de l'île visitée s'adressent aussi aux résidents qui finissent par avoir le bon geste nature.

\subsection{Les gestionnaires du littoral}

Les administrations qui gèrent les littoraux sont nombreuses dans les DROM comme en France métropolitaine, entre les administrations de la mer et celles spécialistes des milieux terrestres. Cependant on peut noter que ces compétences tranchées tendent à se transformer afin de mieux prendre en compte l'étendue de la zone côtière et la nécessité du développement durable.

Les Affaires Maritimes qui s'intéressent traditionnellement à la navigation, aux marinspêcheurs et à la navigation de loisirs, tendent aujourd'hui à élargir leurs compétences lors de l'établissement des Schémas de Mise en valeur de la Mer, des contrats de baie. Elles interviennent afin de valider et de délimiter les zones de cantonnement de pêche et les aires de DCP, ou pour constater les traces de pollution, faire respecter la réglementation de la pêche et surveiller l'intégrité des eaux territoriales. Leur implication dans la gestion des eaux côtières se développe.

Les Directions Départementales de l'Equipement interviennent largement sur les espaces littoraux pour aménager les ports, pour protéger souvent par enrochements les routes littorales et les quartiers vulnérables à l'érosion marine. Elles ont en charge la maîtrise des grands travaux (terminaux portuaires, stations d'épuration), la mise en place des Opérations Programmées d'Amélioration de l'Habitat, les Logements Evolutifs Sociaux qui permettent de résorber les quartiers insalubres et de déplacer ces populations littorales parfois soumises aux aléas de houle cyclonique dans des quartiers en arrière du rivage. Les DDE participent à l'élaboration des Plans Locaux d'Urbanisme et donnent un avis sur les permis de construire en fonction du PLU et du Plan de Prévention des risques. Ainsi les politiques des DDE sont proches de celles des instances politiques : les maires et le département. Elles jouent un rôle important dans l'aménagement des communes quand ces dernières sont petites et ne disposent pas de services d'urbanisme.

Depuis 1975, le Conservatoire du littoral a acquis 875 hectares en Guadeloupe et 1705 à la Martinique et gère en 2009, 1875 hectares en Guadeloupe et 1965 hectares à la Martinique. En effet, depuis 1996, les zones naturelles littorales, sont confiées par arrêté préfectoral au Conservatoire du Littoral. Ces espaces bénéficient alors de mesures de protection et de sauvegarde importantes. Les orientations d'aménagement prises par le Conservatoire du Littoral visent à la protection et à la restauration des écosystèmes afin de faire face aux 
problèmes de déforestation, de surfréquentation et de squattérisassions. L'accueil du public et l'aménagement des sentiers littoraux constituent des objectifs prioritaires. Cependant, la plupart des espaces naturels des 50 pas géométriques remis au conservatoire du littoral a par la suite été confié à l'ONF ou aux Parcs Naturels et aux Réserves qui en assure la gestion.

L'Office National des Forêts gère ainsi 15000 hectares de forêt à la Martinique et depuis 1983, la Forêt Domaniale Littorale qui appartient au domaine privé de l'Etat. Cette forêt couvre les deux tiers de la superficie totale de la zone des cinquante pas géométriques soit 1954 hectares dont 174 sur les îlets. En Guadeloupe, l'ONF gère 1466 ha de FDL et 7455 ha de mangroves. L'Office National des Forêts a une action relativement légère sur les littoraux : mise en place de chemins de randonnée, constructions de carbets pour tenter de fixer les petits marchands de souvenirs ou les petits restaurants, délimitation des aires de parking, mise en place de sentiers de découverte, signalisation des manceliniers (arbuste à la sève toxique).

La DIREN complète l'action du Conservatoire du littoral et assure la cohérence des actions des services de l'Etat dans le secteur de l'environnement, en particulier le Ministère chargé de l'environnement. Elle joue un rôle consultatif et d'expertise lors de la réalisation des PLU, des SAR, SMVM, SDAGE..., prépare et suit l'exécution des actions environnement prévues dans les Plans Etat-Région. Elle veille au respect des lois et des règlements dans les secteurs de l'environnement et participe aussi à la mise en valeur des milieux naturels protégés et remarquables.

L'Ifremer est implanté à la Martinique et son action est multiple : accompagner les marinspêcheurs pour l'amélioration des techniques de pêche, aider les comités locaux des pêches dans le choix de l'implantation des zones de cantonnement. Progressivement l'Ifremer glisse comme les Affaires Maritimes vers l'aménagement intégré des zones côtières.

\subsection{Les élus et les associations militantes}

Les élus et les associations constituent des acteurs très actifs de l'aménagement des littoraux. Dans la plupart des cas, les relations sont tendues entre l'élu qui veut mettre en valeur son bout de plage et l'association. Parfois des convergences s'opèrent quand le développement durable est au cœur du projet municipal. Cependant les associations militantes souvent associées aux mouvements indépendantistes, pêchent par leur extrémisme qui rend parfois leur discours difficile à suivre sur des îles où l'on doit aussi aménager et développer l'économie. Ces associations sont récentes, peu nombreuses et comptent un petit nombre d'adhérents. Il existe près de 50 associations de protection de la nature en Guadeloupe ; mais seulement huit d'entre-elles sont agréées par la préfecture. A la Martinique, l'Assaupamar 
(Association pour la sauvegarde du patrimoine martiniquais), créée en 1986, étiquetée à gauche, proche des mouvements indépendantistes, milite pour un meilleur « ménagement » du territoire martiniquais s'appuyant sur le développement durable. Pour y parvenir l'Assaupamar intervient dans différents domaines qui souvent se mêlent: la conjoncture économique, le problème foncier (aide aux paysans sans titre), l'aménagement routier, la qualité des eaux, les risques naturels, la sauvegarde de la forêt. Sur le littoral, l'Assaupamar dénonce les entorses faites à la loi par différentes municipalités de l'île qui ont des projets touristiques dans des zones de mangrove. Enfin, l'Assaupamar s'attaque, parfois en force, aux grands hôtels qui occupent le haut de plage et qui tentent de privatiser l'accès à la plage, et à certains particuliers (essentiellement les békés et les métropolitains) qui construisent des belles villas en bord de mer, sur les 50 pas géométriques.

Les associations internationales sont aussi présentes comme l'Association Française pour les Récifs Coralliens (ACOR), créée en 1997. Elle compte 130 membres (chercheurs, enseignants, administrateurs, experts, ingénieurs du secteur privé et gestionnaires de récifs coralliens) et a pour objectif de regrouper les spécialistes des récifs coralliens et d'être présente auprès des instances nationales et internationales afin d'y promouvoir les activités de recherche, de protection et de gestion. Le WWF France, centre surtout son activité sur le milieu terrestre mais travaille aussi en partenariat avec les parcs et réserves. Suite à la convention de Carthagène, des associations en réseaux protègent les tortues de la Caraïbe (le WIDECAST). De même les associations de protection des mammifères marins se multiplient dans chaque île.

Si tous les partis politiques font aujourd'hui allusion à l'environnement et aux risques, et cela se comprend dans les petites îles tropicales, certains appuient leur projet de société essentiellement sur le développement durable. On retrouve les partis écologiques traditionnels comme les Verts mais aussi des mouvements proprement antillais comme le Modemas à la Martinique, dont le leader; Garcin Malsa est le maire indépendantiste de Sainte-Anne qui épaule les actions de l'Assaupamar dans ses revendications.

\subsection{Les collectivités territoriales et le défi du développement durable}

Dans les Antilles françaises, les collectivités territoriales ont en charge la gestion du littoral depuis les lois de décentralisation de 1981 et sont confrontées au dilemme d'aménagement entre le développement des infrastructures (portuaires, touristiques, routières) afin de soutenir l'économie et la nécessaire gestion durable. Ces oppositions sont renforcées par l'insularité et 
l'isolement. Afin d'arbitrer les formes d'occupation et d'utilisation des littoraux, ces collectivités ont les compétences pour élaborer les schémas d'aménagement régional.

Les collectivités locales, l'Etat et l'Union Européenne élaborent des plans de développement pour les régions (Programmes Opérationnels Intégrés et contrats de plans) qui ont pour objectif la préservation et l'amélioration du cadre de vie. L'axe environnemental concerne la protection contre les risques naturels et la préservation du patrimoine naturel face à l'urbanisation. Les problèmes de pollution, le traitement des déchets et des eaux usées font aussi l'objet d'une attention particulière. En Guadeloupe, les actions environnementales du Programme Opérationnel Intégré s'élève à 4.5 millions d'euros et permettent de financer le parc national, de créer des réserves naturelles, d'effectuer l'inventaire des ZNIEFF. Des actions sont aussi menées pour protéger le littoral, traiter les eaux et les déchets. A la Martinique, les collectivités financent le Parc Naturel Régional ainsi que la réhabilitation des baies. Elles subventionnent la construction d'émissaires en mer afin d'évacuer les eaux usées de Fort-de-France.

Les maires représentent aussi des acteurs de la gestion du littoral. Cependant, la nécessité du développement durable dépend de la sensibilité des élus qui rencontrent les mêmes dilemmes que les Conseils Régionaux et Départementaux, entre protection et aménagements. Ils ont la maitrise des Plans Locaux d'Urbanisme, des zones constructibles, des zones protégées, des ZNIEFF.

Si les différents acteurs comprennent et défendent la nécessité de l'aménagement durable du littoral, les rivalités entre administrations, universitaires et experts, associations, élus et particuliers sont nombreuses. Chacun, à tous les niveaux estime posséder sa part de vérité et s'approprie l'environnement, s'éloignant alors des principes de la gestion intégrée qui nécessite la collaboration de tous pour tendre vers un objectif commun.

3. De la mise en réserve à la gestion intégrée des littoraux

Depuis les années 1980, les littoraux Guadeloupéens connaissent des dynamiques de gestion particulières. Durant ces années, certains acteurs associatifs, politiques de la CôteSous-le-Vent initient l'écodéveloppement. Il s'appuie sur la gamme des activités traditionnelles : travail du bois, construction de barques saintoises, maraîchage, pêche, polyactivité dans le but de valoriser ces filières et de permettre aux jeunes Guadeloupéens d'envisager de conserver un avenir insulaire. Le Parc Régional de Guadeloupe a joué un rôle moteur dans cette approche. Avec la création de la réserve du Grand-Cul-de-Sac-Marin, une 
dynamique de gestion intégrée est apparue avant l'heure, dès 1987. En effet dans un premier temps, cette réserve est apparue comme un territoire de pêche soustrait aux pêcheurs. Les négociations entre le Parc et les pêcheurs de Port-louis, du Moule, de Sainte-Rose ont permis de réguler la fréquentation en particulier l'utilisation des sennes à colas qui étaient très destructrices pour les récifs coralliens et il en fût de même avec les entreprises touristiques, les clubs de plongées, les croisières découverte de la mangrove et des Depuis la mise en réserve de certains écosystèmes littoraux apparait comme une politique de gestion normalisée et appréciée sur le long terme.

\subsection{Les parcs et les réserves naturelles}

La création des parcs et réserves naturelles apparaissent comme la solution la plus efficace de gestion de certains écosystèmes sensibles, peu anthropisés.

L'archipel de Guadeloupe compte trois réserves naturelles. Les îlets de Petite-Terre de la Désirade sont situés à cinq milles au sud-est de la Pointe des Châteaux. Terre-de-Bas (117 hectares) à l'ouest et Terre-de-Haut (31 hectares) à l'est sont séparées par un étroit chenal de 150 mètres de large et d'une profondeur de six mètres. La réserve naturelle protège les îlets et la zone maritime côtière formée d'herbiers et de récifs coralliens, jusqu'à l'isobathe des 10 mètres. Le Conservatoire du littoral qui en assure la gestion, réglemente la pêche, les activités de découverte ainsi que le mouillage. A la Martinique, des projets de réserve naturelle sont à l'étude après l'acquisition de certains îlets des baies du Robert et du François par le Conservatoire du littoral.

Les cantonnements de pêche complètent l'arsenal des zones protégées. Il s'agit de zones où toute pratique de pêche est interdite en accord avec les Affaires Maritimes et les organisations de professionnels afin de protéger ces aires peuplées de juvéniles. Il y a quatre zones de cantonnement de pêche à la Martinique; il s'agit des baies du Trésor à Trinité et de celle du Robert, de l'îlet à Ramier sur la côte Sud de la baie de Fort-de-France et enfin de la zone comprise entre Sainte-Luce et la Pointe Borgnèse (Le Marin).

En Guadeloupe, les Affaires Maritimes ont défini des réserves marines. La pêche à la senne pour les espèces de type cahut (Harengula humeralis) et quiquia (Decapterus macarellus) ainsi que le cola, le coulirous, le balaou et les orphies peuvent faire l'objet de dérogation après demande auprès de l'administrateur des Affaires Maritimes. Ces réserves marines se localisent à Malendure en Côte-sous-le-vent et dans le Grand-Cul-de-Sac-Marin autour de l'îlet Fajoux et des îlets Christophe, Carénage, la Biche. L'embouchure de la Rivière à Goyave et la pointe à Lambis constituent aussi des réserves marines. 
Enfin la création du Parc Naturel de Guadeloupe et son extension sur le Grand-Cul-de-SacMarin constituent la mise en protection ultime. La Réserve Naturelle du Grand-Cul-de-SacMarin a été créée par décret le 23 novembre 1987. Cette réserve éclatée protège des sites divers : milieux humides du littoral sur une surface de 1600 hectares (mangroves, marais herbacés, forêts marécageuses d'eau douce, prairies et savanes humides) et milieux marins (herbiers, récifs coralliens...) sur une surface de 2100 hectares. L'Office National des Forêts a tout d'abord assuré la gestion de cette réserve qui a été confiée ensuite par l'Etat au Parc National de Guadeloupe depuis 1990.

Depuis 1994, la zone protégée administrée par le Parc National de Guadeloupe est devenue une réserve biosphère gérée localement par le Parc National et par l'UNESCO. Cette réserve biosphère doit remplir trois fonctions : la conservation des écosystèmes et de la biodiversité, le développement économique et humain durable et l'appui logistique pour la surveillance continue, la recherche, l'éducation et l'échange d'informations.

Ces réserves et parcs, constituent des lieux de protection, dans des zones relativement peu soumises aux pressions humaines. Leur succès repose en partie sur la concertation entre les différents usagers, même si certains se sentent encore dépossédés. Cette démarche est aujourd'hui poursuivie sur des espaces densément anthropisés.

\subsection{Les expériences de Gestion Intégrée des Zones Côtières}

La recommandation du Parlement et du Conseil de l'Europe du 30 mai 2002 demandait à chaque état de définir une stratégie nationale. La démarche de GIZC a été officiellement décidée par le Comité interministériel d'aménagement et de développement du territoire du 9 juillet 2001. En 2003, le Comité Interministériel de la Mer met en œuvre la recommandation européenne. C'est dans ce contexte qu'un appel à projet de GIZC est lancé en 2005. 49 dossiers sont remis, dont 4 pour la Guadeloupe et 3 pour la Martinique.

Quatre dossiers sont retenus : celui du Robert à la Martinique et ceux de Terre-de-Bas, Terrede-Haut, Marie-Galante et la Désirade pour la Guadeloupe. Ces trois dossiers séparés constituent en définitive un même projet de GIZC multi-sites. Dans ces îles qui souffrent de la surinsularité, l'enclavement induit de forts taux de chômage et un faible dynamisme économique, mis à part Terre-de-Haut qui est très touristique. Le projet de GIZC relève davantage de l'écodéveloppement, par sa volonté de favoriser l'artisanat (fabrication de salako (chapeau traditionnel), de vêtements...), la pêche, la petite agriculture au profit d'un 
écotourisme à échelle insulaire. L'objectif doit permettre un certain dynamisme économique tout en maintenant la culture et en l'intégrant comme produit d'appel touristique. La partie environnementale n'est guère prise en compte dans ces projets de GIZC, mis à par le projet de réserve halieutique à Marie-Galante et l'amélioration du traitement des déchets domestiques. $\mathrm{Au}$ contraire, le projet de GIZC du Robert intéresse la gestion de la baie qui connaît de multiples dégradations. La baie est profonde de six kilomètres pour deux de large. L'ouverture vers l'Atlantique est limitée par des îlets dont le plus grand; l'ilet Chancel s'allonge sur deux kilomètres. Depuis les années 1980, la population a fortement augmenté, plus de 16\% entre 1990 et 1999 et dépasse 26000 habitants avec plus de 450 habitants/km². Le nombre de logements construit durant cette période passe de 3800 à 6800 . Les constructions individuelles se répartissent sur l'ensemble de la commune, en grande partie sur les pentes et sur quelques pointes littorales (Pointe Savane, Pointe la Rose, Pointe du SableBlanc). Les ensembles collectifs se concentrent autour du bourg mais aussi entre ce dernier et la Pointe Lynch, essentiellement au nord de la baie. On assiste donc à une répartition multipolaire de la population et a certains phénomènes de ségrégation socio-spatiale. Les quartiers résidentiels se démarquent sur les pointes Fort, Savane, Hyacinthe, Sable-Blanc. Sur un total de 391 hectares situés sur les 50 pas géométriques, les constructions couvrent 142 hectares. Ce dynamisme a entraîné une hypersédimentation en fond de baie et une forte dégradation des récifs coralliens.

Grace au programme Liteau2, le site atelier de la baie du Robert se structure en 2003, à l'initiative de J-C. Dao, directeur de la station Ifremer de Martinique, qui constitue une équipe pluridisciplinaire intégrant le Cemagref, le cabinet d'étude Impact Mer, l'Ifremer d'Arcachon, l'Université des Antilles et de la Guyane, de Martinique (Géode, GEREC, CRPLC) et de Guadeloupe (Biologie marine), des doctorants et Universitaires de Toulouse, Bordeaux, Poitiers. Dès les premières réunions, le service environnement et la municipalité du Robert sont associés. La Municipalité a une attente technique : rendre les eaux limpides en fond de baie afin de valoriser les travaux d'embellissement du front de mer.

Après trois années d'études, le bilan de Liteau 2 Martinique reste mitigé. Du côté scientifique, les conclusions renforcent la légitimité de la démarche. Cependant en dehors du microcosme, la notion de GIZC est floue parce qu'elle n'est pas portée par des politiques publiques volontaires. La Municipalité n'a pas mis en place un véritable comité opérationnel de GIZC.

3.3. Les difficultés à intégrer les lieux et les modes de vie 
Les différentes directives insistent sur les étapes de mise place de la GIZC qui nécessitent l'étude multicritère puis l'élaboration du plan de gestion et enfin le suivi et la maîtrise des actions.

\section{Les étapes de la GIZC}

-Un état des lieux sur le temps long, présentant les dysfonctionnements de la zone étudiée. Cet état des lieux doit être approuvé par chacun des acteurs.

-Une réflexion prospective, identification des domaines dans lesquels des choix sont nécessaires et des conflits probables.

-Une phase de concertation permettant de privilégier certains scénarios

-L'élaboration d'un projet commun pour 10 à 20, définissant des objectifs et les indicateurs de résultats associés.

-La mise en place d'un plan de gestion qui définit les actions sectorielles à mener, les objectifs intermédiaires et les indicateurs de gestion.

-Eventuellement la désignation d'une structure opérationnelle ayant la maîtrise des actions à mener.

-La mise en place d'une démarche de suivi et d'évaluation des actions et du plan de gestion.

Rapport français d'application de la Recommandation du Parlement européen et du Conseil du 30 mai 2002 relative à la mise en œuvre d'une stratégie de gestion intégrée des zones côtières en Europe.

L'analyse du travail effectué sur la baie montre plusieurs décalages avec les recommandations de l’Union Européenne. L'unité territoriale de base d'une GIZC correspond au bassin versant. C'est donc une avancée par rapport à une définition du rivage qui ne prend en compte que l'interface terre/mer. Cet espace est pertinent dans le cadre d'études dominées par les sciences environnementales. Cependant la gestion intégrée nécessite de prendre en compte l'emboîtement des échelles et des territoires. Dans le cas des pollutions par exemple l'échelle du bassin versant n'est pas suffisante et d'autres niveaux doivent être pris en compte dans l'étude des polluants. La Martinique est longée par des courants marins en provenance du Sud de l'archipel. En entrant dans la baie du Robert, les eaux de surface contribuent aussi à l'apport de polluants venant du sud de l'île et de l'archipel. De même, le bassin versant comme le territoire communal, administratif, ne constituent pas des échelles d'analyse pertinentes pour appréhender les activités humaines. Le territoire approprié par les marins pêcheurs s'étend en mer et peut varier suivant les saisons et les migrations de poisson. A terre, le territoire fonctionnel déborde la plage d'échouage, pour s'étendre aux quartiers proches. Mais il peut aussi être discontinu et inclure ponctuellement les lieux de vente. De même l'essor des activités de loisirs ne concerne pas uniquement les populations riveraines mais 
l'ensemble des Martiniquais qui fréquentent les lieux avec une fréquence et une intensité variables.

Les trajectoires des mobilités résidentielles s'appréhendent à l'échelle insulaire mais aussi intra communale. Il est en effet très important de connaître les motivations qui expliquent l'installation des nouveaux habitants. De même à l'intérieur de la commune, éxiste-t'il des espaces de rebond dans l'agglomération et des lieux d'habitat idéel vers les pointes ? Les mobilités quotidiennes au sein de l'aire périurbaine ne sont pas abordées pour comprendre les navettes liées aux loisirs, au travail, aux enfants ou pour des raisons commerciales. Ces données permettraient de définir les bassins de vie qui s'étendent entre Le Robert et Fort-deFrance. Ainsi le territoire communal ne constitue pas une échelle d'analyse satisfaisante pour une telle étude. L'échelle d'une zone de GIZC devrait être celle de l'intercommunalité (du Scott par exemple) permettant de prendre en compte une étendue plus cohérente impliquant différents élus afin de pérenniser les expériences et d'impulser une véritable dynamique de gestion.

«La GIZC est un processus de décision fondé sur la connaissance fine des territoires et une observation précise des écosystèmes, une analyse prospective des impacts potentiels des décisions et une évaluation continue de leurs effets ». Cette définition de la DIACT montre l'importance de l'analyse prospective qui n'a pas été abordée. Les municipalités successives ont permis un développement polycentrique et l'ont même accompagné par l'installation de pôles structurants en dehors du centre-ville, comme la zone industrielle de la Semair, l'Ifremer et les immeubles sociaux à la Pointe Lynch. Le PLU prévoit la construction du Centre Caribéen de la Mer à la Pointe Melon, à trois kilomètres du bourg. Trois scénarios sont envisageables. Poursuivre la voie actuelle et conduire à un étalement des activités et des constructions. Développer un modèle polycentrique nécessitant une meilleure distribution des services de proximité ou encore prendre des mesures très restrictives afin de limiter les constructions, avec le risque d'augmenter les prix du foncier et par conséquent d'exclure les populations robertines de souche.

Le rôle des scientifiques et des politiques n'a pas été suffisamment défini et discuté. La direction nationale estime que «l'Ifremer ne fait pas la gestion mais y aide et y contribue » Denis J. «L'Ifremer intervient en appui à la mise en œuvre ou à la préparation de la GIZC en s'inscrivant dans un processus en jeu dans les territoires côtiers. Il répond à la demande des politiques publiques, dès lors qu'une volonté politique est affichée et organisée ». La gestion 
des zones côtières est un acte de politique publique à mettre en œuvre ${ }^{1}$. C'est le politique qui doit impulser sa vision par l'élaboration des documents d'urbanisme et la mise en place d'une réglementation et des mesures coercitives. Dans le cas du Robert, la Municipalité a suivi avec intérêt le travail effectué mais elle n'en était pas le moteur. Dans ces conditions, les conclusions restent des propositions.

Pourtant la notion de l'intégration accompagnant la GIZC constitue pour un élu une formidable opportunité permettant d'impulser de nouvelles formes de gouvernance sur des problématiques d'actualité dans une démarche de démocratie participative associant aux débats les habitants, les professionnels, les associations. Comment penser l'intégration de la commune au reste de la Martinique au niveau économique, résidentiel, environnemental ? Quelle intégration des nouveaux arrivants afin qu'ils s'identifient au territoire et s'y impliquent. Comment intégrer les nouvelles formes d'habiter qui reposent sur des mobilités quotidiennes largement polluantes et émettrices de CO2. Comment diminuer la charge polluante ou sédimentaire sur les pentes, une concertation doit ici prendre naissance entre agriculteurs et nouveaux résidents. Cette problématique touche aussi l'intégration de l'habitat au paysage qui tend à se dégrader.

La gestion intégrée repose aussi sur la didactique des élus mais aussi des scientifiques et des habitants qui ont une mémoire des lieux, et des connaissances vernaculaires et empiriques. Une synergie devrait aboutir de ces deux approches du savoir. Le rapide accroissement de la population induit également des niveaux de connaissance du territoire très différents en fonction de l'année d'installation, des niveaux d'études, des âges, de la capacité de chacun d'investir un lieu. De même, la sensibilité à la dégradation environnementale de la baie dépend aussi du lieu d'habitation. L'information de certains utilisateurs et occupants des pentes fortes, comme des 50 pas géométrique pourrait être renforcée. L'approche didactique doit donc répondre au mieux à ces réalités et permettre de sensibiliser les populations riveraines ou non.

Cette démarche doit aboutir à l'élaboration de projets concrets, de chartes d'actions communes à mettre en œuvre, dans les écoles, les quartiers, les services municipaux...C'est au regard de ces micro projets que l'on pourra expertiser les impacts de cette gestion. C'est dans cette phase que les scientifiques ont toute leur place.

\footnotetext{
${ }^{1}$ Une politique publique se définit comme un enchaînement d'activités, de décisions ou de mesures, cohérentes prises principalement par les acteurs du système politico-administratif d'un pays, en vue de résoudre un problème collectif. Ces décisions, donnent lieu à des actes formalisés, de nature plus ou moins coercitive, visant à modifier le comportement de groupes cibles, supposé à l'origine du problème à résoudre. Corinne Larrue.
} 
Le dossier déposé par la municipalité de Sainte-Anne par le maire indépendantiste et écologiste Garcin Malsa et son équipe n'a pas été validé par le Comité Interministériel de la Mer, pourtant depuis quelques années la population est intégrée à la gestion environnementale communale. L'Union Européenne et l'Etat sont en appui et non à l'initiative de la démarche. Depuis une dizaine d'années, les actions en faveur de la protection du littoral dépassent les aménagements. La commune œuvre pour la création des réserves naturelles des îlets du Cap Chevalier en 1995 et du Cap Ferré en 1996 et pour l'acquisition des marais salants par le Conseil des Rivages d'Amérique en 2001. Elle instaure des journées de sensibilisation: journée «sable propre », collecte sélective des ordures, actions en milieu scolaire. La création d'emplois jeunes pour sensibiliser à la protection de l'environnement se poursuit par la création d'un bureau du patrimoine à la mairie. La municipalité lutte contre les chiens errants sur les plages, contre le camping sauvage et a signé en ce sens une convention avec l'ONF. L'aboutissement de la démarche réside dans le plan de développement durable et solidaire qui prend forme depuis décembre 1998. Un an plus tard, en décembre 1999, la signature d'une convention de transfert de gestion de parcelles situées sur les 50 pas géométriques entre l'Etat et la commune, permet à la municipalité de gérer des zones du Domaine Public Maritime pour une durée de 18 ans. En juillet 2000, après trois ans de réflexion et d'études par la DIREN, 100 millions de francs sont mobilisés durant six ans pour réaliser le plan de développement durable conforté par le SAR. En février 2001, la Ministre de l'Environnement, D.Voynet confirme l'engagement de l'Etat dans ce projet inscrit au Document Unique de Programmation de la Communauté Européenne pour six ans. Le territoire communal est divisé en pôles ayant ses vocations en fonction des activités existantes, de sa fréquentation et de ses traditions : le Cap Chevalier est un pôle centré autour de la pêche et de l'aquaculture, les Salines, zone touristique font l'objet d'une gestion particulière pour l'accueil des visiteurs tout en pensant aux générations futures.

Conclusion

Ces quelques exemples de GIZC, montrent le flou qui entoure ce concept, entre l'écodéveloppement, l'étude scientifique environnementale détachée de l'objectif de gestion et d'intégration des populations, et l'initiative locale qui initie une gestion intégrée de la zone côtière sans l'afficher et sans appartenir au club fermé sélectionné par le Comité Interministériel de la Mer. Pourtant la GIZC est certainement la meilleure manière d'aborder 
la gestion littorale sur le long terme dans ces îles riches, densément peuplées, dotées de législations et de gestionnaires de l'environnement.

Après de nombreuses années de recherche scientifique, les connaissances environnementales suffisent à comprendre le fonctionnement des écosystèmes à des fin de gestion. Les prochains projets devront donc porter davantage sur la mise en place de politiques publiques de gestion intégrée, sur la pédagogie et l'information, sur le changement des comportements des élus comme des particuliers. Cela nécessite peut-être d'envisager un autre mode de vie plus économe d'espace. Les retards dans l'application de la GIZC relève pour beaucoup des élus qui la considère davantage comme une contrainte et non comme un moyen d'articuler les territoires, d'intégrer au projet les nouvelles populations du littoral. L'évolution statutaire qui semble irréversible permettra t'elle une meilleure prise en compte de la population locale dans les projets de gestion ...

\section{Bibliographie}

Angeon, V. et Safache, P. (2008). «Les petites économies insulaires et le développement durable : des réalités locales résilientes ?», Études caribéennes, 11/2008, Petits territoires insulaires et développement durable, [En ligne].

Dao, J-C. (2007). Etude préalable à l'aménagement intégré de la zone côtière : site atelier de la baie du Robert, Rapport final, IFREMER/PRAM, Programme Liteau 2, 62 pages.

Desse, M. et P.Saffache (2005). Les littoraux antillais : des enjeux de l'aménagement à la gestion durable. Ibis Rouge, Paris. 116 pages.

Desse, M. et T. Hartog (dir) (2009). Les littoraux de la Caraïbe, pratiques sociales et nouvelles dynamiques spatiales. Terres d'Amérique $\mathrm{n}^{\circ} 7$, Karthala, Paris. 219 pages.

Diact, Secrétariat général de la mer. (2002). Rapport français d'application de la Recommandation du Parlement européen et du Conseil du 30 mai 2002 relative à la mise en œuvre d'une stratégie de gestion intégrée des zones côtières en Europe. 80 pages.

Dubost, I. Ursulet D. Desse, M. Sélise, M. (2007). Pratiques gestion et représentation du territoire de la baie du Robert. Annexe 1, Programme Liteau 2, IFREMER/PRAM, 65 pages. Guineberteau, T. Meur-Ferec, C et B. Trouillet (2006). «La gestion intégrée des zones côtières en France: mirage ou mutation stratégique fondamental», Vertigo- la revue électronique en sciences de l'environnement, volume 7, numéro 3.

Klein, J. (2003). Protéger le littoral dans les départements français d'outre-mer. Thèse de doctorat, Paris IV, Sorbonne, 2003, 545 pages. 
Meur-Ferec, C. (2007). «La GIZC à l'épreuve du terrain : premier enseignements d'une expérience française. », Développement durable et territoires [En ligne].

Rizand, A. Marie, P et K, Pinte. (2006). « La place des scientifiques dans un processus de gestion intégrée des zones côtières : évaluation des pressions polluantes d'origine terrestre sur la baie du Robert (Martinique) ». Vertigo, vol 7n³,14 pages.

Saffache, P. et F. Nicolas (dir.)(2004). Tourisme et environnement: l'exemple du littoral méridional de la Martinique. Paris: Éditions Publibook Université, Collection Sciences Humaines et Sociales, Série Aménagement et Environnement, 114 pages. 


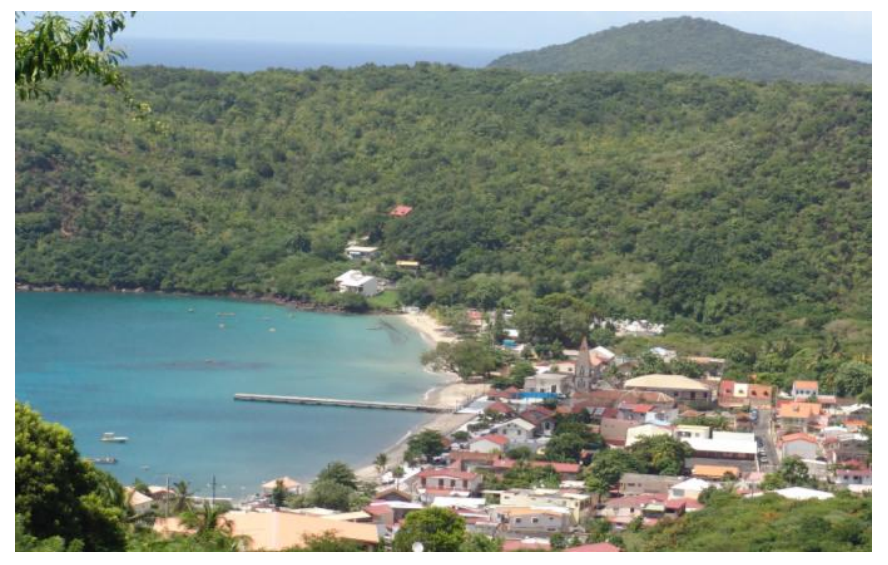

Cliché : B.Gandrille Le bourg des Anses d'Arlets, une littoralisation limitée.

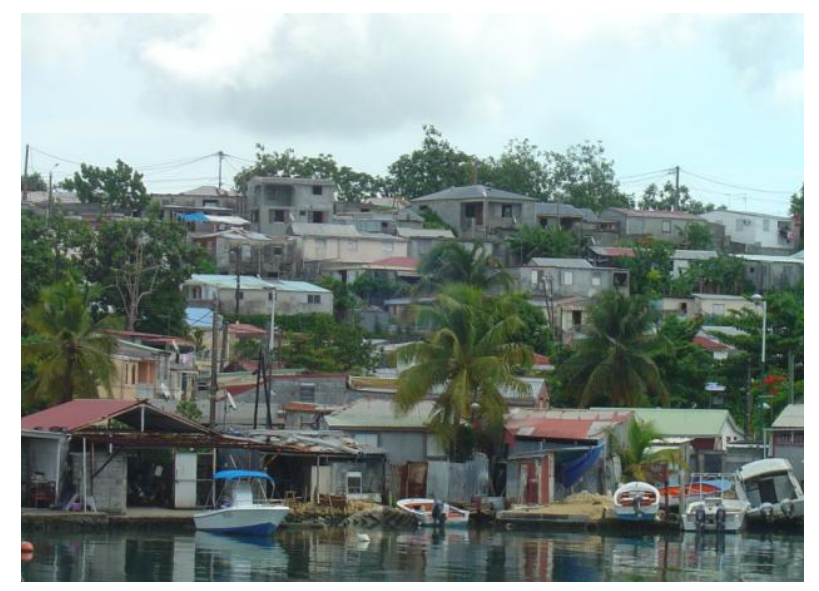

Cliché : M.Sélise Quartier du Carénage à Pointe-à-Pitre, un quartier précaire durcifié 


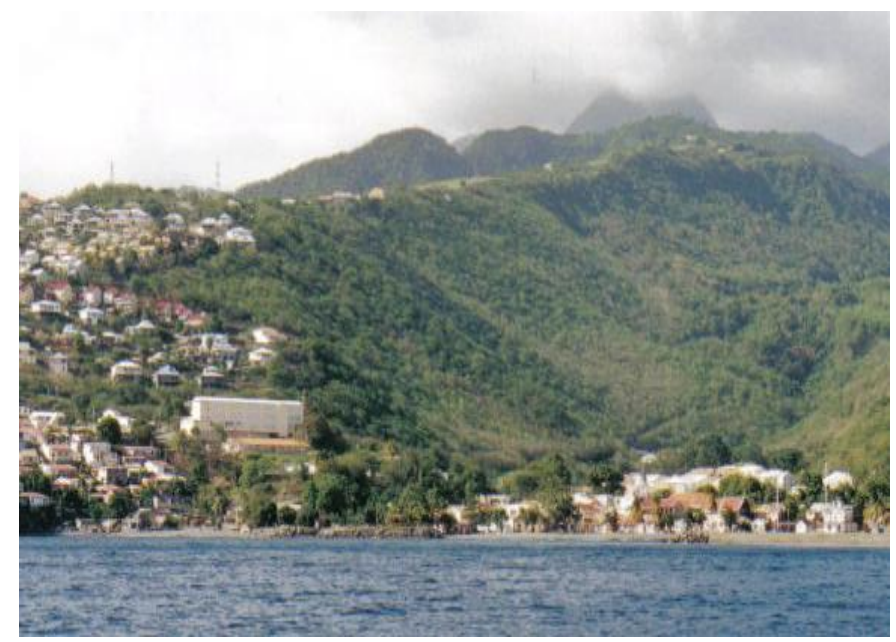

Cliché : M.Desse A droite, au débouché de la vallée, le bourg ancien de Case-Pilote, à gauche, les extensions récentes, maisons modestes en haut de falaise, belles villas sur les pentes et immeuble collectif (HLM). Sur la crête quelques maisons se construisent avec « vue sur mer ».

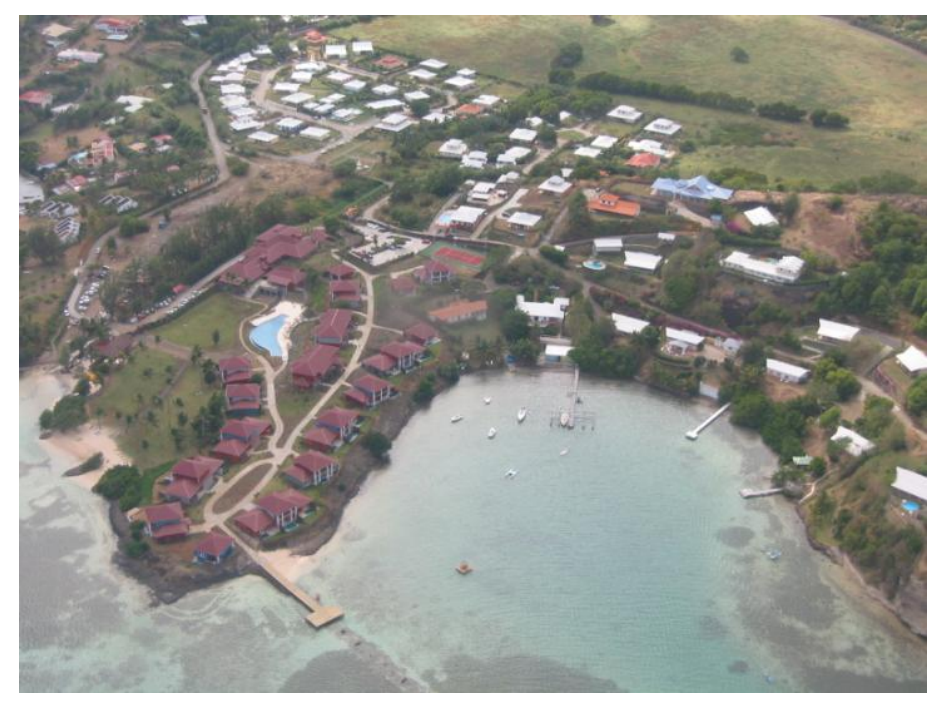

Cliché : J-V Marc. Un quartier résidentiel très récent ou se mêlent, quelques maisons de pêcheurs, des résidences secondaires, un lotissement et le dernier hôtel de luxe, au Vauclin. 